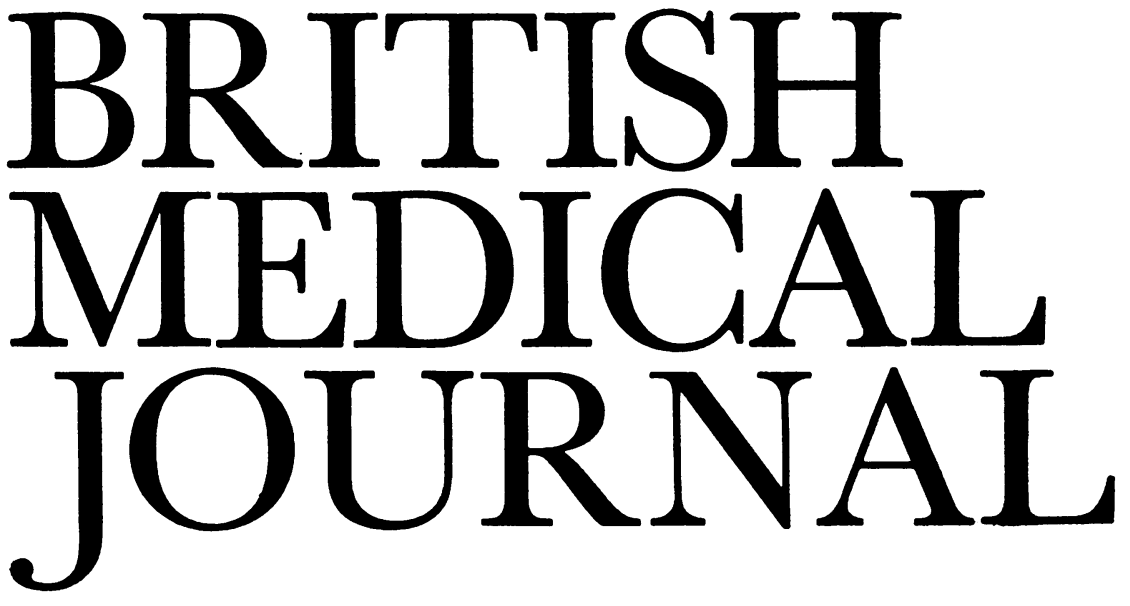

LOEDDN SATURDAY 13 FEBRUARY 1971

\title{
Influenza and the Nervous System
}

Neurological complications such as encephalomyelitis and polyradiculitis may follow childhood exanthemata or accompany specific infections such as infectious mononucleosis. The intractable depression which may sometimes follow attacks of influenza has been attributed to invasion of the brain by the influenza virus, though no direct scientific evidence supports this view.

As noted in these columns last year, ${ }^{1}$ viruses appear to cause neurological symptoms in two main ways. They may be neurotropic, like those of poliomyelitis or herpes zoster or simplex, and produce their effect by direct invasion of the nervous system. Or they may provoke an autoimmune or hypersensitivity response in the nervous system, in which case neurological symptoms tend to appear a few days after the development of the primary illness. The leading article then noted that in that winter's epidemic of influenza some patients had developed neuralgic amyotrophy (shouldergirdle neuritis), others suffering from multiple sclerosis had relapsed acutely, and some other patients had developed symptoms of either encephalitis or encephalopathy, of transverse myelitis, or of an acute peripheral neuropathy resembling the Guillain-Barré syndrome.

Neurological complications of influenza may be more common than many textbooks of neurology would suggest. As W. R. (Lord) Brain and J. N. Walton ${ }^{2}$ pointed out, the difficulty is that influenza is a label often attached randomly to individuals suffering from nonspecific febrile illnesses of short duration, and it may therefore be difficult to confirm the character of the primary illness except by virological studies. No one has yet isolated the influenza virus from the brain of patients suffering from such complications, while on the contrary it is well recognized that invasion of the nervous system by specific viruses can sometimes cause an initial nonspecific febrile illness which is clinically indistinguishable from influenza. Nevertheless, the evidence increasingly suggests that such neurological complications as are seen in confirmed cases of influenza are probably due to an autoimmune or hypersensitivity process precipitated by the presence of the influenza virus in the body but not necessarily in the brain. Thus J. G. Greenfield in $1930^{3}$ described two cases of acute disseminated encephalomyelitis as a sequel to influenza, and similar cases were reported in Great Britain during the pandemic of Asian influenza which occurred in 1957. ${ }^{4-7}$

Now at page 369 of the B.M.F. this week Dr. C. E. C. Wells describes the clinical picture and the results of virological studies carried out in 19 carefully documented cases. All 19 patients suffered upper respiratory infections which could reasonably have been diagnosed as influenza on clinical grounds. Serological studies, however, showed that the infection was probably due to influenza $A$ virus in eight cases and to other viruses (including adenovirus and herpes zoster) in six, while in five cases the studies were negative. It was not possible to isolate a virus or to culture it from the blood in any case. The interval between the onset of the febrile illness and the development of neurological complications varied from one to 112 days, and it was indeed so variable that it is difficult to draw any valid conclusions on whether the neurological state resulted from direct viral invasion of the nervous system or from an autoimmune or hypersensitivity process, though the latter seems more probable. Two patients developed herpes zoster with myelopathy, while six showed a clear-cut picture of transverse myelopathy or myelitis. Four presented with a polyradiculopathy, and four had a combination of neurological signs suggesting lesions of both spinal cord and nerve roots. One patient had encephalopathy and another evidence of disease in both the brain and spinal cord, while the final case developed a subdural empyema which was presumably the result of a superimposed pyogenic infection.

The early symptoms of lesions in the spinal cord were weakness of the legs, retention of urine, and chest pain, and paraesthesiae in the legs were sometimes noted. Total paralysis of the lower limbs sometimes developed within $\mathbf{4 8}$ hours, and a sensory level over the trunk below which sensation was diminished was commonly seen. The cerebrospinal fluid was usually abnormal, having an increase in protein or cells or both. The patients with polyradiculopathy showed a clinical picture characteristic of the Guillain-Barre syndrome, with ascending weakness, distal paraesthesiae, relatively little sensory loss but absent tendon reflexes, slowing of conduction in peripheral nerves, and a rise in cerebrospinal fluid protein. Sometimes the weakness was severe enough to produce 
respiratory paralysis requiring assisted respiration.

In this series of cases the cranial nerves were unusually severely affected, with ophthalmoplegia, facial diplegia, and weakness of bulbar muscles. Occasionally lesions of nerve roots seemed to be restricted and unilateral, giving pain, weakness, and wasting in the muscles of the shoulder girdle on one side, as is well recognized to occur in so-called neuralgic amyotrophy. In two cases severe loss of position and joint sense was noted in both hands, and there was also evidence of spinal cord disease, but there was nothing to indicate from their previous history that these patients were suffering from multiple sclerosis. In a single fatal case there was clinical and pathological evidence of severe lesions of both roots and spinal cord, and the pathological changes were those of a demyelinating radiculomyelitis with some inflammatory cell infiltration. Two patients suffered an encephalopathy with headache and confusion, leading to transient coma or semicoma and producing subsequently a prolonged amnesia for the period of the illness. The cerebrospinal fluid was abnormal in both cases, as was the electroencephalogram.

The author was clearly impressed with the apparent response to corticotrophin by patients in all groups. Certainly the rapid improvement which followed its administration in many of the patients suggested that this remedy was influencing the course of the disease. This response also gives some support to the concept that these complications are due to an autoimmune demyelinating process. On the other hand, spontaneous improvement in transverse myelitis and in the Guillain-Barré syndrome does occur. In the treatment of this syndrome, in particular, neurological opinion is sharply divided at the moment between those who believe that corticotrophin should be given and those who believe that it should not. Majority opinion still appears to favour the view that corticotrophin should be given as early as possible in the course of such illnesses, and Dr. Wells's experience would suggest that it should certainly be given to patients suffering from these rare but increasingly well-recognized complications of influenza and influenza-like illnesses.

${ }^{1}$ British Medical fournal, 1970, 1, 248.

2 Brain, W. R., and Walton, J. N., Brain's Diseases of the Nervous System, 7 th edn. London, Oxford University Press, 1969.

Greenfield, J. G., Fournal of Pathology and Bacteriology, 1930, 33, 453.

Kapila, C. C., Kaul, S., Kapur, S. C., Kalayanam, T. S., and Banerjee, D., British Medical fournal, 1958, 2, 1311.

5 Dunbar, J. M., Jamieson, W. M., Langlands, J. H. M., and Smith, G. H., British Medical fournal, 1958, 1, 913.

6 Flewett, T. H., and Hoult, J. G., Lancet, 1958, 2, 11

7 McConkey, B., and Daws, R. A., Lancet, 1958, 2, 15.

\section{Tanapox}

Tanapox is a newly described disease of exceptional interest. A report on it by Professors A. W. Downie and G. S. Nelson and their colleagues appears at page 363 of the B.M.F. this week. Not only is it caused by a pox virus with unusual characteristics, but the virus is related to a virus known to be oncogenic in man. The disease is probably a zoonosis, and it has occurred in an area where agricultural development and a large increase in human population is foreseen. The Tana River basin (whence the name) in Kenya is a large, mainly arid plain with a small, mostly nomadic population, but plans exist for it to become one of the major areas of irrigated agriculture in Africa.

The disease was first seen in September 1957 as a small outbreak it mission school in the swamp-forest flood plain of the lower reaches of the river. It was again observed as an epidemic of several hundred cases among people living in small groups along the middle reaches of the river between Garissa and Garsen. Both episodes were associated with heavy floods so that the people, their domestic animals, and probably also wild species had to take refuge on "islands" in the flood. Mansonia mosquitoes were breeding extensively in the flood waters. Despite contact between the affected population and the surrounding nomads no spread to the latter was reported, nor did the disease spread to unaffected people in the hospital to which cases were admitted.

Clinically there was fever lasting three or four days, sometimes associated with severe headache, backache, and marked prostration. Patients had one or at most two vesicular lesions which resembled pocks but regressed without pustulation. Lesions occurred on the upper arm, face, neck, or trunk but never on the hands or lower limbs.

The virus isolated from the lesions was shown to be the cause of the disease, because when inoculated into a human volunteer (previously repeatedly vaccinated against smallpox) or into rhesus or vervet monkeys it reproduced the characteristic lesion, from which virus was re-isolated. That the virus was a pox virus was confirmed by the characteristic histology of the lesions, the demonstration of elementary bodies and (by electron microscopy) vaccinia-like particles in them, and, most conclusive, that the virus could reactivate heat-inactivated vaccinia virus. Antiserum raised against the virus in a monkey gave a specific gel-precipitation reaction with extracts from the lesions in the naturally infected patients. The human volunteer had regional lymphadenitis but no systemic illness, perhaps because the virus had been laboratory-passaged.

The unusual features of tanapox virus are that it caused lesions (and probably grows) only in human and monkey cell cultures, not in chick embryos, chick embryo cell cultures, rabbit or bovine embryo cells. It caused no disease in baby mice, guinea pigs, rabbits, a calf, lamb, pig, or goat. No serological relationship could be detected with the vacciniavariola group of pox viruses (which includes monkey pox), with molluscum contagiosum, with a range of domestic animal pox viruses, or with fowlpox virus. However, it showed an antigenic relationship to yabapox virus ${ }^{1}$ and was found to be similar or identical to a "yaba-like" pox virus ${ }^{2}$ which has caused epidemics in at least three primate colonies in the United States. The lesions in the monkeys were similar to those of tanapox and there were no deaths. Nine people handling the monkeys developed lesions on the hand or forearm (usually at sites of injury) ranging in diameter from 1 to $2.5 \mathrm{~cm}$. Some of them had severe lymphangitis, adenitis, and a systemic illness with temperatures over $102^{\circ} \mathrm{F}\left(38.9^{\circ} \mathrm{C}\right) .^{3}$ This "yaba-like" virus is thought to be a latent infection of vervet monkeys or sooty mangabeys, but the outbreaks occurred in macaques. ${ }^{2}$

The relationship of tanapox virus to yaba virus is of interest. The latter was isolated in Nigeria from laboratory rhesus monkeys ${ }^{1}$ with an outbreak of tumours. It has been shown to cause similar benign tumours in man. ${ }^{4}$ It would be useful to know what tanapox virus would do in an immunosuppressed monkey.

The evidence suggests that tanapox is an infection of wild primates transmitted to man mechanically on the contaminated mouth-parts of blood-sucking insects - on the Tana River probably by the flood-enhanced population of Mansonia mosquitoes. Myxomatosis (another pox virus) is similarly transmitted among rabbits-by mosquitoes in Australia but probably mainly by fleas in Britain. In the same way tanapox 